\title{
Polynomial Identities of Banach Algebras
}

\author{
L. Cioletti, J. A. Freitas and D. J. Gonçalves
}

October 22, 2015

\begin{abstract}
In this paper we consider PI-algebras $A$ over the real and complex numbers and address the question of whether it is possible to find a normed PI-algebra $B$ with the same polynomial identities as $A$, and moreover, whether there is some Banach PI-algebra with this property. Our main theorem provides an affirmative answer for this question and moreover we also show the existence of a Banach Algebra with the same polynomial identities as $A$. As a byproduct we prove that if $A$ is a normed PI-algebra and its completion is nil, then $A$ is nilpotent.
\end{abstract}

\section{Introduction}

Polynomial identities algebras or simply PI-algebras has some of its origins in the work of M. Dehn [3] in 1922, motivated by Projective Geometry problems. Later, in 1936, W. Wagner [17] considered some identities for the quaternion algebras, and some authors actually regard this as the first real paper in PIalgebra. As a theory PI-algebras has its starting point in the seminal works of N. Jacobson and I. Kaplansky in the forties ([7], [10]). Comprehensive reviews can be found in the works of Drensky [4], Jacobson [8] and Koshlukov [12].

Nowadays PI-algebra is a classical subject in algebra and a lot of work has been done by exploring some connections with representation theory, graded algebras, Lie algebras and Hopf algebras, see [13, 16, 18]. A PIalgebra which is also a Banach algebra is called Banach PI-algebra. They appeared frequently in some applications and important problems on the area and were recently used to obtain rigorous mathematical results in Quantum information Theory, see [9], where the author used the standard polynomials and the Amitsur-Levitzki theorem and other ideas of polynomial identities to present some effective algorithms for analyzing mathematical properties of quantum channels. 
The aim of this short note is to continue the study initiated in [6], about nilpotency of Banach nil algebras, towards similar results to PI-algebras. The main result of this paper give sufficient conditions for a normed PIalgebra to have the same set of polynomial identities that its completion. As an application of this paper main result's we prove by using the concept of multihomogeneous norm (Definition 1) that if $F\langle X\rangle$ is multihomogeneous normed algebra and $A$ is a PI-algebra such that the completion of the quotient space $F\langle X\rangle / \operatorname{Id}(A)$ is nil, then $A$ is nilpotent. Here $F\langle X\rangle$ denotes the free non-unitary associative algebra, freely generated over $F(\mathbb{R}$ or $\mathbb{C})$ by the infinite set of noncommutative variables $X=\left\{x_{1}, x_{2}, \ldots\right\}$.

We should remark that existence of normed algebra structure on a PIalgebra is a subtle question and it was showed by Dales in [2] the existence of PI-algebras over $\mathbb{R}$ or $\mathbb{C}$ not admitting the structure of a normed algebra. In fact, Dale result's is much more strong as it shows that there exists a nilpotent commutative algebra which is not a normed algebra. To circumvent this obstacle we shown here for any PI-algebra $A$ over $\mathbb{R}$ or $\mathbb{C}$ that there exists a normed PI-algebra $B$ having the same polynomial identities as $A$. So the requirement of the normed algebra structure on PI-algebras treated here does not restrict the applicability of our results.

\section{Preliminaries}

Throughout this paper all the algebras considered will be non-unitary, associative and over the field $F$. Thus for convenience we will only use the term algebra. The key example to keep in mind is the algebra $F\langle X\rangle$. The elements of $F\langle X\rangle$ are called polynomials and a polynomial of the type $x_{i_{1}} x_{i_{2}} \ldots x_{i_{n}}$ is called monomial.

A polynomial $f\left(x_{1}, \ldots, x_{m}\right) \in F\langle X\rangle$ is called a polynomial identity for an algebra $A$ if $f\left(a_{1}, \ldots, a_{m}\right)=0$, for all $a_{1}, \ldots, a_{m} \in A$. We denote by $\operatorname{Id}(A)$ the set of all polynomial identities of $A$. If $\operatorname{Id}(A) \neq\{0\}$ then we say that $A$ is a PI-algebra. The set $\operatorname{Id}(A)$ is an ideal of $F\langle X\rangle$ and has the property that $f\left(g_{1}, \ldots, g_{m}\right) \in I d(A)$ whenever $g_{1}, \ldots, g_{m} \in F\langle X\rangle$ and $f\left(x_{1}, \ldots, x_{m}\right) \in \operatorname{Id}(A)$. This is what is called a $T$-ideal, for details see [4, 5].

Let $F\langle X\rangle^{\left(d_{1}, \ldots, d_{m}\right)}$ be the vector subspace of $F\langle X\rangle$ spanned by all monomials $u=x_{j_{1}} \ldots x_{j_{t}}$, where the variable $x_{i}$ appears $d_{i}$ times in $u$ for all $i=$ $1, \ldots, m$. If $f\left(x_{1}, \ldots, x_{m}\right) \in F\langle X\rangle^{\left(d_{1}, \ldots, d_{m}\right)}$ then we say that $f$ is multihomogenous of multidegree $\left(d_{1}, \ldots, d_{m}\right)$. Note that if $f=f\left(x_{1}, \ldots, x_{m}\right) \in F\langle X\rangle$, we can always write

$$
f=\sum_{d_{1} \geq 0, \ldots, d_{m} \geq 0} f^{\left(d_{1}, \ldots, d_{m}\right)}
$$


where $f^{\left(d_{1}, \ldots, d_{m}\right)} \in F\langle X\rangle^{\left(d_{1}, \ldots, d_{m}\right)}$. The polynomials $f^{\left(d_{1}, \ldots, d_{m}\right)}$ are called the multihomogenous components of $f$.

Now we recall an important result that will be used in the next section, its proof can be found in [5], Theorem 1.3.2.

Theorem 1. If $f=f\left(x_{1}, \ldots, x_{m}\right)$ is a polynomial identity for an algebra $A$, then every multihomogeneous component of $f$ is a polynomial identity for $A$.

We remark that the above result holds for every infinite field $F$, but it is not always true for finite fields.

An algebra $A$ is said to be normed if it satisfies the followings properties: $A$ has a norm $\|\cdot\|$ and $\|a b\| \leq\|a\|\|b\|$ for all $a, b \in A$. It is called Banach algebra if $(A,\|\cdot\|)$ is a complete normed space. Normed algebras have natural structure of metric space, where the distance between $a, b \in A$ is given by $d(a, b):=\|a-b\|$. By considering $A$ as metric space, we can construct another metric space $C(A)$, which contains $A$ as dense subspace and having the following universal property: if $B$ is any complete metric space and $\phi: A \rightarrow B$ is any uniformly continuous function, then there exist an unique uniformly continuous function $\psi: C(A) \rightarrow B$ which extends $\phi$.

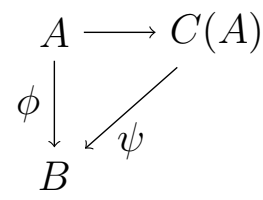

The metric space $C(A)$ is called completion of $A$ and is determined up to isometry by this property, see [11] for more details. In fact, the following strong result holds. Every normed algebra $A$ is contained in some Banach algebra $C(A)$ such that $A$ is dense in $C(A)$.

\section{Main Results}

In this section we state and prove the main result of this paper which is the Theorem 2 .

Proposition 1. If $A$ is a normed PI-algebra, then $\operatorname{Id}(A)=\operatorname{Id}(C(A))$.

Proof. By considering that $A \subseteq C(A)$ we get that $\operatorname{Id}(C(A)) \subseteq \operatorname{Id}(A)$. Conversely, let $f\left(x_{1}, \ldots, x_{m}\right) \in I d(A)$ and $a_{1}, \ldots, a_{m} \in C(A)$. From the construction of $C(A)$ it is easy to see that $f\left(a_{1}, \ldots, a_{m}\right)=0$. The last identity implies that $f \in \operatorname{Id}(C(A))$. Therefore $\operatorname{Id}(A) \subseteq \operatorname{Id}(C(A))$. 
In other words this proposition tell us that every normed PI-algebra $A$ has the same polynomial identities as some Banach PI-algebra. Since not all PI-algebras are normed PI-algebras, see for example [2], a natural question to ask is: given a PI-algebra $A$, is there some Banach PI-algebra $B$ with the same polynomial identities of $A$ ? As commented early we give an affirmative answer for this question and we also show how to construct such Banach PIalgebra $B$. To explain the construction we need to introduce some definitions.

Let $f=f\left(x_{1}, \ldots, x_{n}\right) \in F\langle X\rangle$ be a polynomial, which will be written as

$$
f=\sum_{d_{1} \geq 0, \ldots, d_{n} \geq 0} f^{\left(d_{1}, \ldots, d_{n}\right)}
$$

where $f^{\left(d_{1}, \ldots, d_{n}\right)}=f^{\left(d_{1}, \ldots, d_{n}\right)}\left(x_{1}, \ldots, x_{n}\right)$ is the multihomogeneous component of $f$ with multidegree $\left(d_{1}, \ldots, d_{n}\right)$.

Definition 1. A norm $\|\cdot\|$ in $F\langle X\rangle$ is called multihomogenous if $(F\langle X\rangle,\|\cdot\|)$ is a normed algebra and for all $f \in F\langle X\rangle$ we have $\left\|f^{\left(d_{1}, \ldots, d_{n}\right)}\right\| \leq\|f\|$ for all multidegree $d=\left(d_{1}, \ldots, d_{n}\right)$. If $F\langle X\rangle$ is a normed algebra endowed with a multihomegeneous norm, then we say that $F\langle X\rangle$ is a multihomogeneous normed algebra or simply $M N$-algebra.

Example 1. A multihomogeneous norm can be contructed in $F\langle X\rangle$ in the following way. Given an element $f \in F\langle X\rangle$ consider its standard decomposition $f=\sum_{m} \alpha_{m} m$, where $\alpha_{m} \in F$ and $m$ is a monomial, and then define $\|f\|=\sum_{m}\left|\alpha_{m}\right|$.

We should remark that the requirement that $(F\langle X\rangle,\|\cdot\|)$ is a normed algebra is crucial here and also impose strong restrictions on how to construct multihomogeneous norms. For instance, let $f=\sum_{m} \alpha_{m} m \in F\langle X\rangle$ and $\|\cdot\|$ given by $\|f\|=\sum_{m}\left(1-2^{-1} \delta_{x_{1}}(m)\right)\left|\alpha_{m}\right|$, where $\delta_{x_{1}}(m)$ is equals to one if $x_{1}=m$ and zero otherwise. It is easy to see that $\|\cdot\|$ is indeed a norm on the space $F\langle X\rangle$ but $(F\langle X\rangle,\|\cdot\|)$ is not an normed algebra since $1 / 4=$ $\left\|x_{1}\right\|\left\|x_{1}\right\|<\left\|x_{1}^{2}\right\|=1$. Notice that this norm is just a single modification of the standard sum norm, where the "direction" $x_{1}$ has its scale multiplied by $1 / 2$.

Before we proceed to the statement of the main theorem of this paper we present a very simple auxiliary lemma. On the statement of this lemma we use the following convention: let $g\left(x_{1}, \ldots, x_{t}\right)$ a polynomial and $g^{\left(d_{1}, \ldots, d_{t}\right)}$ its multihomogeneous component of multidegree $\left(d_{1}, \ldots, d_{t}\right)$. If $m<t$ and $d_{m+1}=d_{m+2}=\ldots=d_{t}=0$, then we write $g^{\left(d_{1}, \ldots, d_{m}, \ldots, d_{t}\right)}=g^{\left(d_{1}, \ldots, d_{m}\right)}$. If $t<m$, then we write $g^{\left(d_{1}, \ldots, d_{t}\right)}=g^{\left(d_{1}, \ldots, d_{t}, 0, \ldots, 0\right)}$, where the number of zeros is $m-t$. 
Lemma 1. Let $(F\langle X\rangle,\|\cdot\|)$ be a MN-algebra and let $f=f\left(x_{1}, \ldots, x_{m}\right)$ be a polynomial. If $\left\{f_{n}\right\}_{n \in \mathbb{N}}$ is a sequence in $F\langle X\rangle$ such that $f_{n} \rightarrow f$, then $f_{n}^{\left(d_{1}, \ldots, d_{m}\right)} \rightarrow f^{\left(d_{1}, \ldots, d_{m}\right)}$ for all multidegree $d=\left(d_{1}, \ldots, d_{m}\right)$.

Proof. Since $\|\cdot\|$ is a multihomogeneous norm in $F\langle X\rangle$, we have from the definition the following inequality $\left\|f_{n}^{\left(d_{1}, \ldots, d_{m}\right)}-f^{\left(d_{1}, \ldots, d_{m}\right)}\right\| \leq\left\|f_{n}-f\right\|$. So the convergence of $f_{n}^{\left(d_{1}, \ldots, d_{m}\right)} \rightarrow f^{\left(d_{1}, \ldots, d_{m}\right)}$ is an immediate consequence of the convergence

$f_{n} \rightarrow f$.

The next is the main theorem of this work and it provides the construction of the Banach PI-algebra $B$ mentioned above.

Theorem 2. Let $F\langle X\rangle$ be a multihomogenous normed algebra. If $A$ is a PI-algebra, then $\operatorname{Id}(A)=\operatorname{Id}(C(F\langle X\rangle / \operatorname{Id}(A)))$.

Proof. Let $A$ be a PI-algebra. We claim that if $F\langle X\rangle$ is a MN-algebra, then $\operatorname{Id}(A)$ is a closed ideal. In fact, let $\left\{f_{n}\right\}_{n \in \mathbb{N}}$ in $\operatorname{Id}(A)$ be a sequence of polynomials such that $f_{n} \rightarrow f$. We want to prove that $f \in \operatorname{Id}(A)$. Write

$$
f=\sum_{\left(d_{1}, \ldots, d_{m}\right)} f^{\left(d_{1}, \ldots, d_{m}\right)}
$$

By the Lemma 1, we have that $f_{n}^{\left(d_{1}, \ldots, d_{m}\right)} \rightarrow f^{\left(d_{1}, \ldots, d_{m}\right)}$ for all multidegree $\left(d_{1}, \ldots, d_{m}\right)$. Note that $f_{n}^{\left(d_{1}, \ldots, d_{m}\right)} \in F\langle X\rangle^{\left(d_{1}, \ldots, d_{m}\right)} \cap I d(A)$ by the Theorem 1 .

Since $F\langle X\rangle^{\left(d_{1}, \ldots, d_{m}\right)}$ is a finite-dimensional vector space follows that the intersection $F\langle X\rangle^{\left(d_{1}, \ldots, d_{m}\right)} \cap I d(A)$ has also finite dimension. Since every finitedimensional space is closed in the norm topology, we have immediately that $F\langle X\rangle^{\left(d_{1}, \ldots, d_{m}\right)} \cap I d(A)$ is closed. Thus $f^{\left(d_{1}, \ldots, d_{m}\right)} \in F\langle X\rangle^{\left(d_{1}, \ldots, d_{m}\right)} \cap \operatorname{Id}(A)$ and therefore $f \in \operatorname{Id}(A)$. The multihomogeneous norm in $F\langle X\rangle$ induces a norm in the quotient algebra $F\langle X\rangle / I d(A)$ given by $\|f+I d(A)\|=\inf \{\|f+g\|$ : $g \in I d(A)\}$, where $f \in F\langle X\rangle$. It is simple to verify that the quotient $F\langle X\rangle / \operatorname{Id}(A)$ endowed with this norm is also a normed algebra. From a classical result in PI-algebra we get that $\operatorname{Id}(A)=\operatorname{Id}(F\langle X\rangle / \operatorname{Id}(A))$, see [5]. So the proof of the theorem follows immediately from Proposition 1.

Remark 1. Some results from associative PI-algebras can be extended to nonassociative algebras, see $[1,15]$. For instance, if $F\langle X\rangle$ is a nonassociative algebra, then $\operatorname{Id}(A)=\operatorname{Id}(F\langle X\rangle / \operatorname{Id}(A))$. But in the definition of Banach algebras one needs the associativity. 


\section{Applications}

As mentioned before, Grabiner proves in [6] for a Banach algebra $A$ that if $A$ is nil then $A$ is nilpotent. As an application of the Theorem 2 we obtain similar results for PI-algebras. In Grabiner's theorem, the algebra $A$ is required to be a Banach algebra, whilst here we investigate when the nilpotency of an algebra $A$ can be deduced from $C(A)$. In this direction our first result is

Corollary 1. Let $A$ be a normed PI-algebra. If $C(A)$ is nil, then $A$ is nilpotent.

Proof. If $C(A)$ is nil, it follows from Grabiner's theorem that $C(A)$ is nilpotent. Thus $x_{1}^{n}$ is a polynomial identity of $C(A)$ for some $n$. From the Proposition 1 we have $\operatorname{Id}(A)=\operatorname{Id}(C(A))$, so $A$ is nilpotent.

In the next application we do not assume that $A$ is normed algebra and then we obtain the nilpotency of $A$ from the properties of the completion of a certain quotient space related to the polynomial identities of $A$.

Corollary 2. Let $(F\langle X\rangle,\|\cdot\|)$ be a multihomogenous normed algebra and let $A$ be a PI-algebra. If $C(F\langle X\rangle / I d(A))$ is nil, then $A$ is nilpotent.

Proof. By taking $B=F\langle X\rangle / I d(A)$ we get from the Grabiner theorem that $C(B)$ is nilpotent since we are assuming that $C(B)$ is nil. Thus $x_{1}^{n}$ is a polynomial identity of $C(B)$ for some $n$. By the Theorem 2 we have $\operatorname{Id}(A)=$ $\operatorname{Id}(C(B))$, therefore $A$ is nilpotent.

Acknowledgements. L. Cioletti was supported by FEMAT, J. A. Freitas and D. J. Gonçalves were supported by grant from CNPq No. 478318/2010-3 and No. 480139/2012-1.

\section{References}

[1] Y. Bahturin, Identical relations in Lie algebras, VNU Scienc Press BV, Utrecht, First English Edition, 1987.

[2] H. G. Dales, Norming Nil Algebras, Proc. Amer. Math. Soc., 83, Number $1,71-74,1981$.

[3] M. Dehn, Über die Grundlagen der projektiven Geometrie und allgemeine Zahlsysteme, Math. Annalen, 1, 184-194, 1922. 
[4] V. Drensky, Free algebras and PI-algebras, Graduate Course in Algebra, Springer, Singapore, 1999.

[5] A. Giambruno, M. Zaicev, Polynomial identities and asymptotic methods, Math. Surveys Monographs 122, AMS, Providence, RI, 2005.

[6] S. Grabiner, The nilpotency of Banach nil algebras, Proc. Amer. Math. Soc., 21, 510, 1969.

[7] N. Jacobson, Theory of Rings [Russian translation]. Izd. Inostr. Lit., Moscow, 1947.

[8] N. Jacobson. PI-algebras. Ring theory Proc. Conf., Univ. Oklahoma, Norman, Okla., 7, 1-30, 1973.

[9] A. Jamiołkowski, On applications of PI-algebras in the analysis of quantum channels, Int. J. Quantum Inf., 10, Number 8, 1-13, 2012.

[10] I. Kaplansky, Rings with a polynomial identity. Bull. Amer. Math. Soc., 54, 575-580, 1948.

[11] I. Kaplansky, Set Theory and Metric Spaces, Allyn and Bacon Series in Advanced Mathematics, Boston, Mass., 1972.

[12] P. Koshlukov, Algebras with polynomial identities. Mat. Contemp., 16, 137-168, 1999.

[13] A. I. Malcev, On algebras defined by identities (Russian), Mat. Sb. 26, 19-23, 1950.

[14] M. A. Naimark, Normed algebra, Wolters-Noordhoff, 3 edition, 1972.

[15] L. H. Rowen, Polynomial identities in Ring Theory, Academic Press, New York, 1980.

[16] W. Specht, Gesetze in ringen, Mathematische Zeitschrift, 52, 557-589, 1950.

[17] W. Wagner, Über die Grundlagen der projektiven Geometrie und allgemeine Zahlensysteme, Math. Ann., 113, 528-567, 1937.

[18] S. Yu. Vasilovsky, The basis of identities of a three-dimensional simple Lie algebra over an infinite field, Algebra and Logic 28, 355-368, 1989. 
Departamento de Matemática UNIVERSIDADE DE BRASÍlIA Brasília, BraziL cioletti@mat.unb.br jfreitas@mat.unb.br

Departamento de Matemática Universidade Federal de São Carlos, São Carlos, Brazil dimas@dm.ufscar.br 